\title{
Dissecting mitosis with laser microsurgery and RNAi in
}

\section{Drosophila cells}

\author{
António J. Pereira ${ }^{1}$, Irina Matos ${ }^{1}$, Mariana Lince-Faria ${ }^{1}$ and Helder Maiato ${ }^{1,2,{ }^{*}}$ \\ ${ }^{1}$ IBMC - Instituto de Biologia Molecular e Celular, Universidade do Porto, Rua do Campo Alegre 823, \\ 4150-180 Porto, Portugal \\ ${ }^{2}$ Lab. Cell and Molecular Biology, Faculdade de Medicina, Universidade do Porto, 4200-319 Porto, \\ Portugal \\ * Corresponding author
}

Running head: Laser microsurgery in Drosophila

Key Words: Mitosis; Drosophila; S2 cells; HeLa cells; Live Cell Microscopy; RNAi; Agar Overlay; Laser microsurgery

\section{Contact:}

Helder Maiato

Instituto de Biologia Molecular e Celular,

Universidade do Porto

Rua do Campo Alegre, 823

4150-180 Porto

Portugal

Tel: +351-22-6074900

Fax: +351-22-6099157

E-mail: maiato@ibmc.up.pt 


\section{Abstract}

Progress from our present understanding of the mechanisms behind mitosis has been compromised by the fact that model systems that were ideal for molecular and genetic studies (such as yeasts, C. elegans or Drosophila) were not suitable for intracellular micromanipulation. Unfortunately, those systems that were appropriate for micromanipulation (like newt lung cells, PtK1 cells or insect spermatocytes) are not amenable for molecular studies. We believe that we can significantly broaden this scenario by developing high resolution live cell microscopy tools in a system where micromanipulation studies could be combined with modern gene-interference techniques. Here we describe a series of methodologies for the functional dissection of mitosis by the use of simultaneous live cell microscopy and state-of-the-art laser microsurgery, combined with RNA interference (RNAi) in Drosophila cell lines stably expressing fluorescent markers. This technological synergism allows the specific targeting and manipulation of several structural components of the mitotic apparatus in different genetic backgrounds, at the highest spatial and temporal resolution. Finally, we demonstrate the successful adaptation of agar overlay flattening techniques to human HeLa cells and discuss the advantages of its use for laser micromanipulation and molecular studies of mitosis in mammals. 


\section{Introduction}

During every cell cycle the genetic information must be replicated in order to be equally distributed in the form of chromosomes to the progeny cells at the time of mitosis. In the last two decades mitosis research has attracted unprecedented attention because of its fundamental relationship to the etiology of cancer. Nevertheless, despite enormous recent progress, a solid view for how chromosomes are segregated during cell division remains elusive. It is, however, well established that the remarkable movements of chromosomes during division are mediated and monitored by the kinetochore, a minute structure that forms the interface between chromosomes and a microtubule-based apparatus known as the mitotic spindle (1).

Over the last years laser microsurgery has been established as one of the most important tools to investigate how mitosis works. The mitotic apparatus is an appealing context for the use of such spatial domain techniques because it contains discrete structures that can be manipulated in order to address their respective roles in the production of pulling or pushing forces to move chromosomes during mitosis. Accordingly, laser microsurgery has been seminal to elucidate the mechanistic basis of the spindle-assembly checkpoint (2), the role of the centrosome in spindle assembly (3) and the role of kinetochores in chromosome movement (4-6). We believe that we can significantly broaden this scenario by combining powerful live cell imaging and state-of-the-art laser technology with molecular tools. Recent advances in the study of how genes function in living cells, when combined with increasingly sophisticated lasers and microscopes with higher spatial (nanometers) and temporal (milliseconds) resolutions, represents the next new challenge for cell surgeons and will provide a powerful approach to unravel the molecular mechanisms behind many concurrent processes that drive mitosis. 
Here we propose the systematic use of Drosophila melanogaster somatic cells in culture to perform intracellular micromanipulation towards a functional dissection of mitosis. We envision that questions regarding acentrosomal spindle formation, the spindle checkpoint signalling mechanism, the role of kinetochore-microtubule dynamics for chromosome movement, as well as the fate of cells with a compromised mitosis could be addressed by the synergistic combination of laser microsurgery and RNAi tools. Indeed, this approach has already been successfully used in Drosophila S2 cells to investigate the role of kinetochores in spindle morphogenesis (7), as well as to elucidate the molecular mechanism of spindle microtubule flux at kinetochores (8).

As a first step towards the application of laser microsurgery to Drosophila it would be required the establishment of cell lines stably expressing different GreenFluorescent Protein (GFP) and/or red variant fluorescent-tagged components of the mitotic apparatus (e.g. microtubules, centrosomes, kinetochores and chromosomes) $(9,10)$. By combining Differential Interference Contrast (DIC) and epi-fluorescence microscopy one will be able to image at least 2 of those constituents simultaneously in living cells.

Drosophila provides several key advantages for cell division studies. First, the genome is fully sequenced and more than $60 \%$ of Drosophila proteins are conserved with humans (11). More relevant, $75 \%$ of genes associated with human diseases have orthologues in Drosophila (12). Second, Drosophila has only 4 pairs of chromosomes (in comparison with 23 pairs in the case of human cells), and individual kinetochore-microtubule fibers (K-fibers) can be easily observed at the light microscopy (LM) level. Third, Drosophila is one of the most powerful genetic systems known, with an accumulated knowledge extending more than 100 years of 
research and extensive collections of mutants for genes involved in mitosis are freely available. Fourth, protein redundancy at the cellular level is minimal in Drosophila, whereas humans for example, usually express several isoforms or closely related proteins. Fifth, specific gene silencing can be easily achieved by RNA interference (RNAi), by simply adding dsRNA to the culture medium (13). This is particularly useful for high-throughput genome-wide screenings, which are now well established (14-16). One major disadvantage though, has been related with the fact that most Drosophila cell lines are semi-adherent and thus cells are round, making highresolution microscopy difficult. To overcome this problem we have modified the agaroverlay technique recently described for Drosophila culture cells by Fleming and Rieder (2003)(17) so that we can control the degree of cell flattening. Unlike cells flattened by growing on a concanavalin A substrate (18), which is particularly useful for routine microscopy studies, the agar overlay approach allows one to select cells that are sufficiently flattened to be imaged without compromising the ability of cells to progress through mitosis and undergo normal cytokinesis. By taking advantage of stable cell lines expressing fluorescent components of the mitotic apparatus, and by focusing high energy pulses of laser light through a high resolution lens, it would be possible to selectively destroy, cut and otherwise manipulate K-fibers, chromosomes, kinetochores and centrosomes in living cells and in different genetic backgrounds.

To conclude, we discuss the possibility of extending some of the abovementioned methodologies to human cells in culture, particularly the adaptation of the agar overlay technique to HeLa cells. This will improve the resistance and viability of mitotic cells during 3D time-lapse recordings by reducing the phototoxic effects of light and also stabilize the focal plane throughout mitosis. This will facilitate 
the use of RNAi studies combined with laser micromanipulation during mitosis in a mammalian model system.

\section{Materials}

\subsection{Cell culture}

Drosophila Schneider 2 cells (see Note 1):

1. Disposable $25 \mathrm{~cm}^{2}$ tissue culture flasks and/or 6 well plates

2. Heat-inactivated Fetal Bovine Serum (FBS) (GIBCO \#10500-064)

3. Schneider's Drosophila medium (Sigma \#S0146)

4. Disposable sterile pipettes $(1,5,10$ and $25 \mathrm{ml})$

Human HeLa cells (see Note 1):

1. Dulbecco's Modified Eagle's Medium (DMEM) (GIBCO \#11880) supplemented with $10 \%$ of heat inactivated FBS.

2. Sterile Phosphate Buffered Solution (PBS) $\mathrm{pH} \mathrm{7,4}$

3. $0.05 \%$ Trypsin, $0.53 m M$ EDTA (Trypsin-EDTA, GIBCO \#25300)

4. Disposable $25 \mathrm{~cm}^{2}$ tissue culture flasks (T-flasks) and/or 6 well plates

5. Disposable sterile pipettes $(1,5,10$ and $25 \mathrm{ml})$

\subsection{Stable transfections of S2 cells}

1. S2 cells exponentially growing in 6 well plates $\left(1 \times 10^{6}\right.$ cells $\left./ \mathrm{ml}\right)$ (see Note 2$)$

2. Serum Free Schneider's Drosophila medium (SFM) 
3. Complete Schneider's Drosophila medium (Schneider's medium supplemented with $10 \%$ of heat-inactivated FBS)

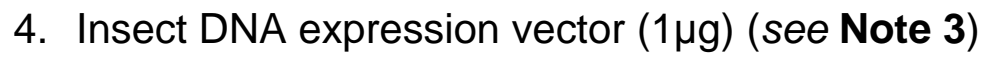

5. Sterile $1.5 \mathrm{ml}$ microcentrifuge tubes

6. Cellfectin insect transfection reagent (Invitrogen \#10362-010)

7. Selection antibiotics (Hygromycin-B, Sigma \#H3274 or Blasticidin S HCl Fluka BioChemika \#15205)

\subsection{RNAi in S2 cells}

1. S2 cells exponentially growing in 6 well plates $\left(10^{6}\right.$ cells $\left./ \mathrm{ml}\right)$

2. Schneider's Drosophila SFM

3. Complete Schneider's Drosophila medium

4. Target DNA specific PCR primers containing the T7 RNA polymerase-binding site (TAATACGACTCACTATAGGG)

5. Linear DNA fragments approximately 700 bp in length (obtained by PCR from the target cDNA or genomic DNA)

6. PCR Clean-Up kit (MoBio Laboratories, Inc. \#12500-100) (see Note 4)

7. MEGAscript T7 Kit (Ambion \#AM1334)

\subsection{Live cell microscopy}

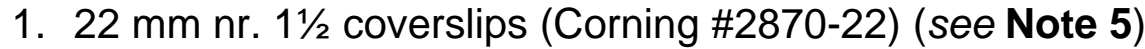

2. $25 \mathrm{~mm} \mathrm{nr} .1 \frac{1}{2}$ coverslips (Corning \#2870-25)

3. Modified Rose chambers (ref) (see Note 6)

4. $5 \mathrm{ml}$ syringes 
5. $26 \mathrm{G}$ needles

6. Low melting agarose (SIGMA \#A-9414)

7. Complete Schneider's Drosophila medium (for S2 cells).

8. Leibovitz's L-15 Medium without phenol red (GIBCO \#31415) supplemented with $10 \%$ FBS (for HeLa cells) (see Note 7)

9. Modified Rose chambers

10. Concanavalin A coated coverslips (for S2 cells) (see Note 8)

11. Poly-L-lysine coated coverlips (for HeLa cells) (see Note 9)

\subsection{Laser microsurgery}

1. Nd:YAG laser $2^{\text {nd }}$ harmonic $(532 \mathrm{~nm})$ single-mode, 8-ns pulses (ULTRA-CFR TEM00 Nd:YAG from Big Sky Laser - Quantel). Laser pulse transverse profile approximates the fundamental (Gaussian) mode (M-squared factor $\cong 1.5$ ).

2. Attenuating stage comprising a half-wave plate and a polarizing cube (CVI Corporation, QWPM-532-05-2-532 and PBSO-532-050) and a beam dump.

3. Pulse energy fine-tuning stage comprising a half-wave plate (CVI Corporation, QWPM-532-05-2-532) mounted on a rotating stage and a $\lambda / 8$ plane glass window at Brewster's angle (aprox. 56 ${ }^{\circ}$ ) and a beam dump.

4. Beam expander (LINOS Photonics) with variable magnification (2-8x) tuned to match the laser beam diameter to the objective pupil diameter.

5. Half wave plate (Thorlabs, WPMH05M-532) used to align the polarization axis of the beam with one of the principal axis of the DIC Wollaston prism. 
6. Periscope (2 mirrors).

7. Dichroic mirror (Semrock, FF493/574-Di01): reflective at $532 \mathrm{~nm}$ and transmissive at the GFP emission spectral window.

8. Nikon TE2000U microscope with 'stage-up kit'.

\section{Methods}

\subsection{Cell culture}

Drosophila S2 cells grow at $25^{\circ} \mathrm{C}$ in Schneider's complete medium.

Human HeLa cells grow at $37^{\circ} \mathrm{C}$ with $5 \% \mathrm{CO}_{2}$, in DMEM medium supplemented with $10 \%$ of heat-inactivated FBS.

\subsection{Establishment of stable S2 cell lines expressing fluorescently- tagged proteins}

\section{Day 1: Preparation of cells}

1. Prepare exponentially growing $\mathrm{S} 2$ cells for transfection by seeding $10^{6} \mathrm{cells} / \mathrm{ml}$ in a 6 well plate in $2 \mathrm{ml}$ complete medium.

Day 2: Transient transfection (see Note 10)

2. Prepare the following transfection mix (per well) just before transfection:

Solution A: $1 \mu \mathrm{g}$ of the plasmid DNA 
$25 \mu$ of SFM

Solution B: $5 \mu$ l of Cellfectin

$25 \mu$ of SFM

3. Slowly add solution A dropwise to solution B with continuous mixing and leave at room temperature for $45-60 \mathrm{~min}$.

4. Centrifuge the cells at $1000 \mathrm{rpm}$ for $5 \mathrm{~min}$ and replace the cell culture medium with $450 \mu$ of fresh SFM.

5. Mix the solution prepared in 2 and add dropwise to each well. Swirl to mix in each drop.

6. Incubate $3-4$ hours at $25^{\circ} \mathrm{C}$.

7. Replace the SFM with complete medium after washing once.

8. Incubate for 1-2 days.

\section{Day 4: Selection (Stable transfection) (see Note 10)}

9. Add $1 \mathrm{ml}$ of fresh complete medium and start the selection using the appropriate selection antibiotic, starting with the abovementioned concentrations. Replace the medium every 4-5 days until resistant cells start expanding. Note that it is normal that those cells that do not incorporate the plasmid will die (see Note 11).

\section{+2-3 Weeks: Expansion}


10. Centrifuge cells and resuspend in complete medium containing the appropriated selection agent. Passage cells at a 1:2 dilution when they reach a density of 6 to $20 \times 10^{7}$ cells $/ \mathrm{ml}$. When cells become more concentrated they may be passed at higher dilutions, typically 1:5 or 1:10.

11. Expand resistant cells into $25 \mathrm{~cm}^{2}$ flasks and test for expression of the protein of interest by western blot and/or fluorescence microscopy.

\subsection{RNAi}

\section{Preparation of dsRNA}

1. Design 18-mer sequence-specific oligonucleotides to make a PCR product of $\sim 700 \mathrm{bp}$ from the cDNA or genomic DNA of interest (see Note 12). Don't forget to incorporate the 5' T7 RNA polymerase binding site into your primers.

2. Prepare $10-12$ individual $P C R$ reactions on ice containing the following reagents:

- $\quad$ 0.5-1 ng of template cDNA from a plasmid or 0.5-1 $\mu$ g of genomic DNA

- $1 \mu \mathrm{M}$ of each primer

- $0.2 \mathrm{mM}$ of dNTP mix

- Taq polymerase enzyme buffer

- Enzyme Buffer

- $2 \mathrm{mM} \mathrm{MgCl}_{2}$ (if not included in the enzyme buffer)

- water to a final volume of $100 \mu \mathrm{l}$ (take in consideration the volume of Taq polymerase that will be added afterwards) 
3. Set up the following PCR program (this may have to be adjusted to your specific conditions):

- $94^{\circ} \mathrm{C}-2 \min$

- add $2.5 \mathrm{U}$ of Taq polymerase to the PCR reaction

- $94^{\circ} \mathrm{C}-30 \mathrm{sec}$

- $55^{\circ} \mathrm{C}-60 \sec \times 30$ cycles

- $72^{\circ} \mathrm{C}-60 \mathrm{sec}$

- $72^{\circ} \mathrm{C}-10 \min$

4. Purify the PCR products by pooling 3 reactions, and running them through each column from a PCR Clean-Up Kit according to the manufacturer's instructions.

5. Test $1 \mu \mathrm{l}$ of the clean product by electrophoresis on a $1 \%$ agarose gel and quantify the DNA by measuring $A b s_{260}$. The DNA yield is calculated as follows:

$\mathrm{Abs}_{260} \times$ dilution factor $\times 50=$ DNA conc. in $\mu \mathrm{g} / \mathrm{ml}$

6. Use this DNA as template for at least 10 in vitro RNA synthesis reactions with the MEGAscript T7 Kit (Ambion) according to the manufacturer's instructions, except that the incubation time should be increased to at least $6 \mathrm{~h}$ (see Note 13).

7. Pool the reactions into a single tube and precipitate the RNA with $\mathrm{LiCl}$ and RNAse free water according to the instructions included with the kit. 
8. Carefully wash the pellet once with $70 \%$ ethanol and let it air-dry.

9. Re-suspend the pellet in $100 \mu \mathrm{l}$ of nuclease-free water and check the RNA concentration as before but using the following algorithm:

Abs $_{260} \times$ dilution factor $\times 40=$ RNA conc. in $\mu \mathrm{g} / \mathrm{ml}$

10. Denature RNA secondary structures by heating at $65^{\circ} \mathrm{C}$ for $30 \mathrm{~min}$ on a beaker containing $200 \mathrm{ml}$ of previously warmed water and then let it cool down slowly to room temperature to make dsRNA duplexes.

11. Test $1 \mu \mathrm{l}$ of the dsRNA by electrophoresis in $1 \%$ agarose gel. It should run like DNA as a clean band of $\sim 700 \mathrm{bp}$. Sometimes a lower band is also visible. This corresponds to ssRNA that did not annealed into duplexes.

12. Store the dsRNA at $-20^{\circ} \mathrm{C}$.

\section{Live cell imaging of depleted cells}

1. Grow Drosophila S2 cells in T-flasks at $25^{\circ} \mathrm{C}$ in Schneider's complete medium for 4 days.

2. Check how many time points (how many wells) will be needed for the experiment and whether an efficient depletion requires a $2^{\text {nd }}$ pulse of dsRNA.

3. Distribute $1 \mathrm{ml}$ of serum free media containing $10^{6}$ cells per each well

5. Add $30 \mu \mathrm{g}$ of specific dsRNA to half of the wells, and an equivalent amount of control dsRNA to the other half. Mix well by swirling and leave the cells at $25^{\circ} \mathrm{C}$ for $1 \mathrm{~h}$ to allow incorporation of the dsRNA. 
6. Add $2 \mathrm{ml}$ of complete Schneider's Drosophila medium to each well and put the cells back in the incubator at $25^{\circ} \mathrm{C}$.

7. At the specific time point use a cell scraper to collect an S2 cell suspension for microscopy analysis and also take a sample for western blot (typically $10^{6}$ cells per time point) to monitor knockdown efficiency.

\subsection{Flattening cells for live cell microscopy}

S2 cells (concanavalin A) (see Note 14)

1. Place $22 \times 22 \mathrm{~mm}$ coverslips previously coated with concanavalin $A$ in the bottom of a 6 -well plate. Put $300 \mu$ of an S2 cell suspension on top of the coated coverslip and allow cells to adhere to the glass at $25^{\circ} \mathrm{C}$ for 2 hours.

2. Mount modified Rose chambers and observe under the microscope.

\section{S2 cells (agar overlay)}

1. Prepare a $170 \mu \mathrm{m}$ thick layer of agarose as following (Figure $1 \mathrm{~A}$ ):

i) Place two rectangular coverslip fragments (obtained from a $25 \times 25 \mathrm{~mm}$ coverslip) on opposite ends of a slide, to act as spacers. Put a drop of PBS or water to stick the spacers with the slide (prepare another spare slide that will be required on step 3 , but using $1 / 4$ of a $25 \times 25 \mathrm{~mm}$ coverslip as spacers and without sticking them to the slide).

ii) Using a heater, melt $0.1 \mathrm{~g}$ of low melting agarose in $5 \mathrm{ml}$ of Schneider's SFM. 
iii) After heating, supplement the mixture with $10 \%$ FBS and pipette the liquid agarose into the space between the coverslip fragments.

iv) Place another slide on top of the agarose to form a sandwich and wait until solidifies. This can be kept at $4^{\circ} \mathrm{C}$ for a week in a humid chamber.

v) Carefully separate the two slides with a razor blade.

2. Cut a $1 \times 1 \mathrm{~cm}$ piece from the agarose layer and gently place it on top of a drop of an S2 cell suspension that was previously placed on a $25 \times 25 \mathrm{~mm}$ coverslip.

3. Carefully invert the coverslip containing the cells on agarose onto the new slide (from 1i) (see Note 15)

4. Seal the lateral edges of the coverslip/slide preparation with warm VALAP (1:1:1 vaseline:lanolin:paraffin) to prevent evaporation and observe under the microscope (Figure 2).

\section{HeLa cells (agar overlay)}

HeLa cells are one of the most popular model systems for the study of mitosis in humans. However, their round morphology during mitosis makes them unsuitable for several microscopy studies like those involving fluorescence and DIC. This problem may be overcome by increasing the number of optical sections through a thick volume (typically 10-12 $\mu \mathrm{m}$ ), which implies higher exposure to light. In order to overcome this problem we have adapted our agar overlay protocol to HeLa cells (Figure 1B), which reduces the thickness to $4-5 \mu \mathrm{m}$, resulting in 2-3x decrease in light exposure when fluorescence is used. On the other hand, when only DIC is 
required, the agar overlay reduces cell movement and maintains cells in the same focal plane over time as they progress through mitosis (Figure 3).

1. Grow HeLa cells in DMEM supplemented with $10 \% \mathrm{FBS}$ at $37^{\circ} \mathrm{C}$ with $5 \% \mathrm{CO}_{2}$ until they reach $60-80 \%$ confluence.

2. Remove growth medium and wash adherent cells with PBS pre-warmed at $37^{\circ} \mathrm{C}$ to remove FBS.

3. Add $1 \mathrm{ml}$ of trypsin-EDTA and incubate at $37^{\circ} \mathrm{C}$ for $5 \mathrm{~min}$.

4. Ressuspend detached cells and transfer the supernatant to a tube with $2 \mathrm{ml}$ of media with FBS (to inactivate trypsin). Place a poly-L-lysine treated 22x22 mm coverslip to each $35 \mathrm{~mm}$ plate (or to each well of a 6-well plate) and seed $4 \times 10^{5}$ cells per well in DMEM supplemented with FBS. Allow cells to adhere by growing for $18-24 \mathrm{~h}$ at $37^{\circ} \mathrm{C}$ with $5 \% \mathrm{CO}_{2}$.

5. Prepare a $170 \mu \mathrm{m}$ thick layer of agarose as described in point 1ii of the agar overlay protocol for S2 cells but replacing Schneider's SFM by Leibovitz's L15 SFM.

6. Mount the coverslip with adherent cells in an open Rose chamber and add a small drop of L-15 supplemented with $10 \% \mathrm{FBS}$. Cut a $1 \times 1 \mathrm{~cm}$ piece from the agarose layer and gently place it on top of the cells.

7. Carefully add enough L-15 supple FBS to the cells just to prevent dehydration but without detaching the Agar.

8. Close the chamber with a clean $22 \times 22 \mathrm{~mm}$ coverslip and observe the cells by DIC + fluorescence at $37^{\circ} \mathrm{C}$. 


\subsection{Laser microsurgery}

1. The set-up used for laser microsurgery is designed to ablate intra-cellular structures at the sub-micron level. The schematic representation of the components of the workstation is shown in detail in Figure 4. We use an infinity-conjugated Nikon Eclipse TE2000-U microscope with a 'stage-up kit' and an extra filter turret (Figure 5) added in the plane-wave region of the microscope (between the objective and the tube lens), which allows the injection of external, collimated, light beams into the microscope's light path.

2. We use $532 \mathrm{~nm}$ nano-second pulses to perform microsurgery. This option is mainly driven by the fact that similar pulse parameters have been used successfully in the past $(9, \mathbf{1 0})$. It should be stressed however that, given the non-resonant character of the light-matter interaction processes relevant to microsurgery, other wavelengths, as well as other pulse-widths, may be used (19).

3. A number of laser beam properties have to be modulated before steering into the microscope body, namely the beam diameter, energy, polarization and pointing direction. Our laser is much more energetic (about 1000-fold) than needed (1-2 $\mu \mathrm{J})$, so we used a first strong attenuating stage composed of a half-wave plate and a polarizing cube. We have set this attenuation to approximately its maximum value (but not quite, as the maximal extinction regime may normally introduces aberrant phase profiles) and this is used as a constant attenuator in our set-up. These two components are subjected to the 
highest irradiance in the set-up: it should be guaranteed that the damage threshold of the optics coatings is not exceeded.

4. The second stage is used for fine-tuning the pulse energy. The polarizing cube is here substituted by a plane, uncoated, glass plate with surface quality $\lambda / 8$ at the Brewster's angle, where polarization selection is highest. This is much cheaper than the polarizing cube and serves perfectly the purpose of varying beam attenuation upon half-wave plate rotation.

5. Beam expansion is performed using a variable expander although a custom 2-lens system may be used if the needed magnification is constant. Matching the beam diameter to the objective pupil diameter allows usage of the full numerical aperture of the objective, hence minimizing laser spot size: the expected diameter, $d$, for a (non-truncated) Gaussian beam and high-quality focusing optics is approximated by $d \approx \lambda / N A$, where $\lambda$ is the wavelength inside the medium and NA is the numerical aperture of the objective. On the other hand, excessive expansion leads to strong truncation of the laser beam (at the objective pupil) which may introduce diffraction rings at possibly significant levels. However, if the diffraction rings are kept weak, it may be preferable to introduce them than losing focusing capacity of the central diffraction disk. The focal plane of the laser should coincide (within a fraction of the wavelength) with the focal plane of the imaging system; this can be guaranteed either by axial translation of the CCD or of the second lens of the beam expander.

6. Beam diameter, along with beam quality, is determinant to approach diffraction-limited ablation. A typical bulk measure of beam quality is the M- 
squared parameter, which for our laser is approximately 1.5 . This roughly means that the minimal spot diameter is increased by $50 \%$. For lower-quality beams, spatial filtering should be considered to block propagation of highorder (non-gaussian) beam modes.

7. Before steering the beam into the microscope body, a half-wave plate is used to align the polarization axis of the beam with one of the principal axes of the objective DIC crystal. This prevents the beam to be split into two 'DIC-like' beams.

8. Adjustment screws are used for optical alignment. Mirror $\mathrm{M}_{2}$ and the periscope $\left(M_{3}\right.$ and $\left.M_{4}\right)$ are translated to align the beam in the $X$ and $Y$ axis of the objective's back-focal plane (BFP), respectively. Mirror $M_{4}$ tilt adjustment allows the alignment of the beam in the $X$ and $Y$ axis of the focal (sample) plane. Operationally, apart from casual alignments, we maintain mirror $\mathrm{M}_{4}$ steering the beam roughly to the center of the field of view, and use the XYstage to move the sample and choose the ablation target. In our particular case, we define a $512 \times 512$ pixels region-of-interest in the CCD centered in the actual beam spot. Fine-tuning the CCD region-of-interest center instead of carefully aligning the beam with the microscope optical axis is perfectly acceptable for small beam deviations.

9. The laser beam is steered into the microscope optical axis through a dichroic mirror with the convenient spectral features: reflective at the laser line and transmissive at the GFP spectral window. Our particular dichroic mirror also has the following positive and negative features: i) it is reflective at the $480 \mathrm{~nm}$ region, which allows the use of this spectral region for GFP point-bleaching 
laser injection; and ii) it is not highly transmissive in the emission spectral region of TexasRed and similar fluorescent markers (see Note 16). In summary, if only the microsurgery $532 \mathrm{~nm}$ laser is to be coupled to the microscope, we advise the use of a 'laser-line' dichroic mirror, i.e., one that reflects in the 532 region and is transmissive otherwise (see Figure 6 for examples of laser microsurgery).

10. Operationally, we use Nikon's NIS-Elements to control the whole system with the exception of the laser itself, which is controlled through a custom routine written in Labview where the laser pulse train properties are set, namely the number of pulses (typ, 1-5 pulses), the pulse train frequency (typ. 20Hz) and the pulse energy (typ. 1-2 $\mu \mathrm{J}$ ).

\section{Notes}

1. Ideally tissue culture should be performed without the use of antibiotics, which may select for particular resistant sub-clones. Nevertheless, if the conditions for cell culture are not ideal, especially regarding Drosophila S2 cells that do not require sophisticated environmental control, the use of an antibiotic/antimycotic cocktail is recommended (Penicillin G + Streptomycin sulphate +Anphotericin B; Sigma \#A5955)

2. Exponential growth of Drosophila S2 cells is usually achieved 2-3 days after each passage.

3. We have been successfully using pAc5.1/V5-His A, B, C (Invitrogen \# V411020) and pMT/V5-His A, B, C (Invitrogen \# V412020) for constitutive or inducible expression of fluorescent fusion proteins, respectively. 
4. Alternatively, it is possible to gel-purify the PCR products using a gel extraction kit (Qiagen \#28704).

5. Most objective lens of the main microscope manufacturers are infinity corrected assuming a glass coverslip with $0.17 \mathrm{~mm}$ thickness. This thickness corresponds to coverslip nr. $1 \frac{1}{2}$ (range from 0.16 to $0.19 \mathrm{~mm}$ ).

6. Rose chambers are a proven, cost-effective alternative for re-usable chambers for live-cell imaging. We designed our own modified version of Rose chambers taking into account our specific objective features and to allow different assembly options (e.g. open or closed chambers). Nevertheless, there are other possible commercial alternatives. We recommend the MatTek glass bottom culture disks (MatTek \#P35G-1.5-20-C). The same principles discussed previously regarding coverslip thickness also apply in this case.

7. Phenol red is a common $\mathrm{pH}$ indicator used in cell culture media that may interfere with live cell microscopy fluorescent assays. Therefore, it is highly recommended the use of media without this dye that are usually available from most manufacturers.

8. For coating with concanavalin A, immerse acid-treated coverslips into a concanavalin A (Calbiochem \#2345467) solution $(0.5 \mathrm{mg} / \mathrm{ml})$. Carefully place the coverslips against the sides of a Petri dish forming $\sim 45^{\circ}$ angles, with filter paper at the bottom and let them air-dry. Before using the coated coverslips, put them under UV light for 20 min. 
9. For coating with poly-L-lysine, place a 1:10 dilution of a poly-L-lysine solution $0.1 \% \mathrm{w} / \mathrm{v}$ (Sigma \#P8920) in PBS on top of coverslips and leave it at room temperature for $1 \mathrm{~h}$. Wash extensively with PBS. Carefully place the coverslips against the sides of a Petri dish forming $\sim 45^{\circ}$ angles, with filter paper at the bottom and let them air-dry. Before using the coated coverslips, put them under UV light for 20 min.

10. In order to generate a stable cell line, we highly recommend transfecting exponentially growing S2 cells with a single expression vector that includes both the antibiotic resistance gene for selection of transfected cells and the gene of interest. In the lab we have been using either Hygromycin (300 $\mu \mathrm{g} / \mathrm{ml})$ or Blasticidin $(25 \mu \mathrm{g} / \mathrm{ml})$ with $100 \%$ success in several different cell lines. It is also possible to obtain stable transfections by using two separate vectors, one expressing the antibiotic resistance gene and the other expressing the gene of interest. However, the efficiency drops considerably, given that not all the antibiotic resistant cells will be expressing the gene of interest.

11. Drosophila S2 cells grow better if kept concentrated in culture. However, after antibiotic selection of transfected cells, the vast majority of cells die and survivor cells take a long time to recover. In order to overcome this problem, it is recommended to change the selection medium with antibiotic for fresh complete medium after the first day of selection. Allow cells to recover for 2-3 days and re-start the selection protocol.

12. In some cases, RNAi efficiency increased when this PCR product covered the codon of the first methionine but we and others have successfully knockdown genes using a PCR product exclusively covering the 5' untranslated region. 
Importantly, it is highly recommended to perform BLAST searches with the corresponding target sequence to rule out the possibility of off-target effects.

13. Work in an RNAse free environment. The major source of RNAse is usually the operator, hence it is highly recommended the use of gloves.

14. Note that this type of coating is known to interfere with cytokinetic furrow ingression.

15. It is important to note that less fluid produces greater flattening, but also reduces viability over time. The ideal situation is when one leaves only enough media to make contact with only one of the spacers. As the result cells closer to the dry spacer remain relatively rounded, while cells closer to the wet spacer became extremely flat. This approach allows the formation of a gradient of flatness to select those cells that were ideal for high-resolution light microscopy analyses, but were not inhibited in their mitotic progression or cytokinesis.

16. It should be noted that although the $532 \mathrm{~nm}$ laser only ablates material within a well-defined region (once the pulse energy is properly tuned), the beam profile at the focal plane necessarily spreads out to distant regions and may be enough to bleach fluorescent markers that absorb (even if slightly) in the green region, like mRFP, mCherry and many spectrally similar markers (20). In those cases where dual-color imaging is needed, other laser wavelengths should be considered to perform the microsurgery. 


\section{Acknowledgements}

We are greatly indebted to Alexey Khodjakov and Valentin Magidson for their invaluable advice regarding the development of our laser microsurgery system. We would also like to thank all the colleagues working with Drosophila S2 cells, especially Gohta Goshima, Monica Bettencourt-Dias and Mar Carmena, for sharing materials and for their intellectual contribution related with the development and optimization of the techniques described in this paper. I.M. and M.L-F. respectively hold a Ph.D. studentship (SFRD/BD/22020/2005) and a Post-doctoral fellowship (SFRH/BPD/26780/2006) from Fundação para a Ciência e a Tecnologia of Portugal. The set up of the laser microsurgery system was supported by grants from LusoAmerican Foundation (L-V-675/2005), Crioestaminal/Viver a Ciência and the Gulbenkian Programmes for Research Stimulation and Frontiers in the Life Sciences. Work in the lab. of H.M. is supported by grants POCI/SAUMMO/58353/2004 and PTDC/BIA-BCM/66106/2006 from Fundação para a Ciência e a Tecnologia of Portugal (POCl2010 and FEDER).

\section{Figures}

Figure 1 - Illustration of the agar overlay flattening procedure. (A) For slide preparations two $1 / 4$ fragments of a $25 \times 25 \mathrm{~mm}$ coverslip are cut with a diamond pen (1). These fragments will work as spacers (2). A drop of cell suspension is on a $25 \times 25 \mathrm{~mm}$ coverslip (3). A small agarose square piece (4) is placed on top of the cells sitting on the coverslip (5), which is then carefully flipped $180^{\circ}$ and positioned in contact with one of the spacers on the slide (6). The medium should only make contact with one of the spacers to generate a gradient of flatness (7). With the help 
of a cotton bud the coverslip edges are sealed with warmed VALAP (8). When it solidifies the chamber is ready for observation (9). (B) For Rose chambers, HeLa cells are grown on a $22 \times 22 \mathrm{~mm}$ poly-L-lysine coverslip and placed on the flat surface from the chamber part that will be proximal to the objective (1). A square piece of the agarose layer is positioned on top of the cells (2), followed by the silicon part of the chamber (3). The top part of the chamber that will be closer to the condenser is put with the flat surface in contact with the silicon (4). A small piece of filter paper is then soaked with medium to prevent fast evaporation (5) and the chamber is closed by placing another $22 \times 22 \mathrm{~mm}$ coverslip in the top part of the chamber (6). Finally, four stainless steel bolts are used to seal the chamber (7).

Figure 2 - The agar overlay technique in Drosophila S2 cells. (A) Time lapse sequence of control and (B) CLASP-depleted S2 cells expressing GFP-a-tubulin and DIC. Time in min:sec. Scale bar is $5 \mu \mathrm{m}$.

Figure 3 - Adaptation of the agar overlay technique to human HeLa cells. (A) Time lapse sequence of a HeLa cell growing on poly-L-lysine coated coverslips, without agar, at the metaphase-anaphase transition. (B) Time lapse sequence of a HeLa cell growing on poly-L-lysine coated coverslips, with agar overlay, at the metaphaseanaphase transition. (A', B') Higher magnification views from the same cells shown in $A$ and $B$. The focal plane was kept at a fixed position in each case throughout the experiment. Note that mitotic cells filmed under the agar-overlay remained focused and fairly immobile, while mitotic cells without agar were highly mobile and chromosomes quickly went out of focus after anaphase onset. As a reference, a nucleus/nucleolus from a neighboring interphase cell is shown for each situation. Time is shown in min:sec. Scale bar is $10 \mu \mathrm{m}$. 
Figure 4 - Optical setup for laser microsurgery and imaging.

Figure 5 - Laser microsurgery workstation. (A) General picture of the $1 \times 1 \mathrm{~m}$ workstation with the environmental control chamber. (B) and (C) General view of the external optics that control and steer the laser beam into the microscope. (D) Laser source.

Figure 6 - Examples of laser microsurgery in Drosophila S2 cells stably expressing GFP-a-tubulin. (A) Laser microsurgery on a kinetochore-fiber in a metaphase spindle from S2 cells. The unstable, newly exposed, microtubule plus-ends prompt full depolymerization of the kinetochore-fiber at a very high rate $(\sim 20 \mu \mathrm{m} / \mathrm{min})$.

Horizontal bars: $1 \mu \mathrm{m}$, vertical bar: $10 \mathrm{sec}$. (B) Similar experiment in another cell. The target is in this case more distant to the chromosome, leaving a small portion of the microtubule fiber attached to the kinetochore. In addition to the rapid catastrophe on the pole-directed fiber portion, polymerization is observed from the kinetochore at approximately $1.4 \mu \mathrm{m} / \mathrm{min}$. Horizontal bar: $1 \mu \mathrm{m}$, vertical bar: 30sec. (C) Laser microsurgery on an interphase microtubule. Horizontal bar: $1 \mu \mathrm{m}$.

\section{References}

1. Maiato, H., J. DeLuca, E.D. Salmon, and W.C. Earnshaw (2004) The dynamic kinetochore-microtubule interface. J Cell Sci. 117, 5461-77.

2. Rieder, C.L., R.W. Cole, A. Khodjakov, and G. Sluder (1995) The checkpoint delaying anaphase in response to chromosome monoorientation is mediated by an inhibitory signal produced by unattached kinetochores. J Cell Biol. 130, 941-8.

3. Khodjakov, A., R.W. Cole, B.R. Oakley, and C.L. Rieder (2000) Centrosomeindependent mitotic spindle formation in vertebrates. Curr Biol. 10, 59-67.

4. Brenner, S., D. Pepper, M.W. Berns, E. Tan, and B.R. Brinkley (1981) Kinetochore structure, duplication, and distribution in mammalian cells: 
analysis by human autoantibodies from scleroderma patients. J Cell Biol. 91, 95-102.

5. Khodjakov, A. and C.L. Rieder (1996) Kinetochores moving away from their associated pole do not exert a significant pushing force on the chromosome. $\mathrm{J}$ Cell Biol. 135, 315-27.

6. Khodjakov, A., R.W. Cole, A.S. Bajer, and C.L. Rieder (1996) The force for poleward chromosome motion in Haemanthus cells acts along the length of the chromosome during metaphase but only at the kinetochore during anaphase. J Cell Biol. 132, 1093-104.

7. Maiato, H., C.L. Rieder, and A. Khodjakov (2004) Kinetochore-driven formation of kinetochore fibers contributes to spindle assembly during animal mitosis. J Cell Biol. 167, 831-40.

8. Maiato, H., A. Khodjakov, and C.L. Rieder (2005) Drosophila CLASP is required for the incorporation of microtubule subunits into fluxing kinetochore fibres. Nat Cell Biol. 7, 42-7.

9. Khodjakov, A., R.W. Cole, and C.L. Rieder (1997) A synergy of technologies: combining laser microsurgery with green fluorescent protein tagging. Cell Motil Cytoskeleton. 38, 311-7.

10. Magidson, V., J. Loncarek, P. Hergert, C.L. Rieder, and A. Khodjakov (2007) Laser microsurgery in the GFP era: a cell biologist's perspective. Methods Cell Biol. 82, 239-66.

11. Adams, M.D., et al. (2000) The genome sequence of Drosophila melanogaster. Science. 287, 2185-95.

12. Fortini, M.E., M.P. Skupski, M.S. Boguski, and I.K. Hariharan (2000) A survey of human disease gene counterparts in the Drosophila genome. J Cell Biol. 150, F23-30.

13. Maiato, H., C.E. Sunkel, and W.C. Earnshaw (2003) Dissecting mitosis by RNAi in Drosophila tissue culture cells. Biol Proced Online. 5, 153-161.

14. Boutros, M., et al. (2004) Genome-wide RNAi analysis of growth and viability in Drosophila cells. Science. 303, 832-5.

15. Echard, A., G.R. Hickson, E. Foley, and P.H. O'Farrell (2004) Terminal cytokinesis events uncovered after an RNAi screen. Curr Biol. 14, 1685-93.

16. Goshima, G., et al. (2007) Genes required for mitotic spindle assembly in Drosophila S2 cells. Science. 316, 417-21.

17. Fleming, S.L. and C.L. Rieder (2003) Flattening Drosophila cells for highresolution light microscopic studies of mitosis in vitro. Cell Motil Cytoskeleton. 56, 141-6.

18. Rogers, S.L., G.C. Rogers, D.J. Sharp, and R.D. Vale (2002) Drosophila EB1 is important for proper assembly, dynamics, and positioning of the mitotic spindle. J Cell Biol. 158, 873-84.

19. Berns, M.W. (2007) A history of laser scissors (microbeams). Methods Cell Biol. 82, 1-58.

20. Shaner, N.C., R.E. Campbell, P.A. Steinbach, B.N. Giepmans, A.E. Palmer, and R.Y. Tsien (2004) Improved monomeric red, orange and yellow fluorescent proteins derived from Discosoma sp. red fluorescent protein. Nat Biotechnol. 22, 1567-72. 
A

\section{Control}
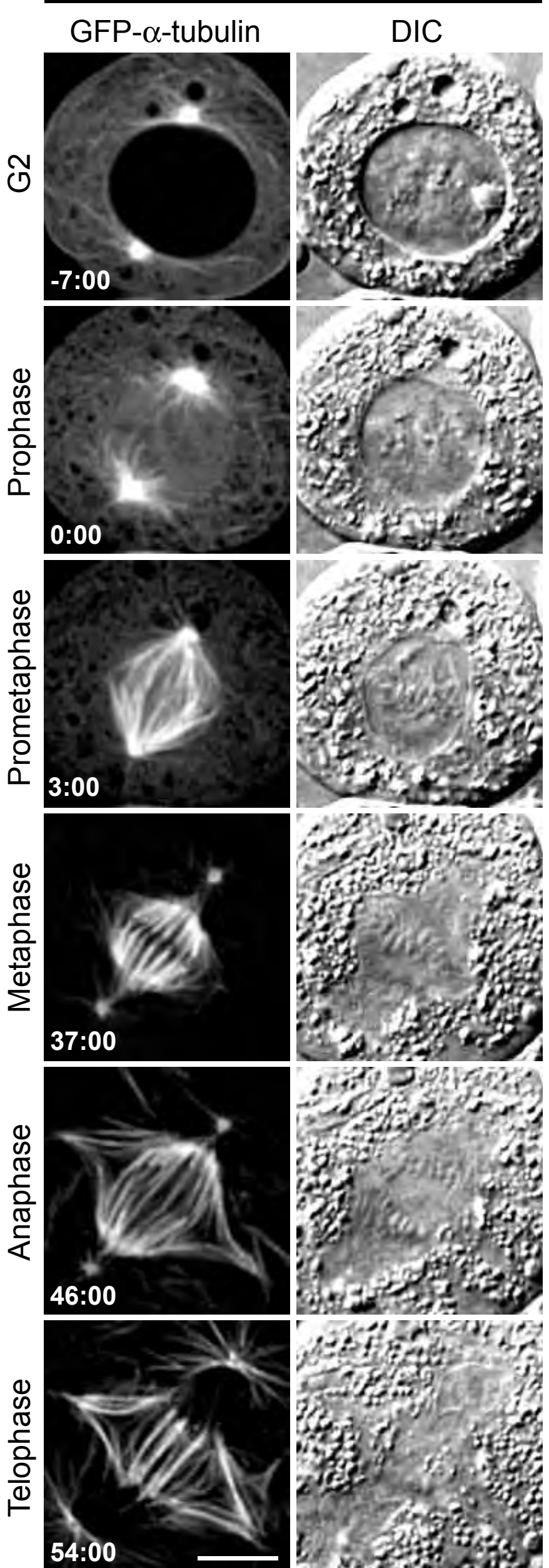

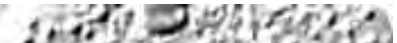

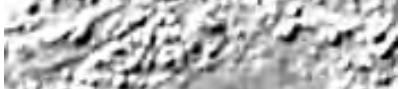
2.3: of 1 in 132 90 ay als

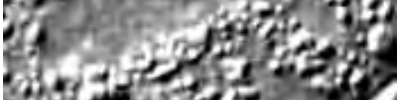

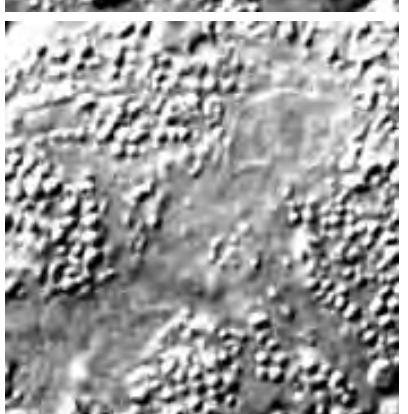

B

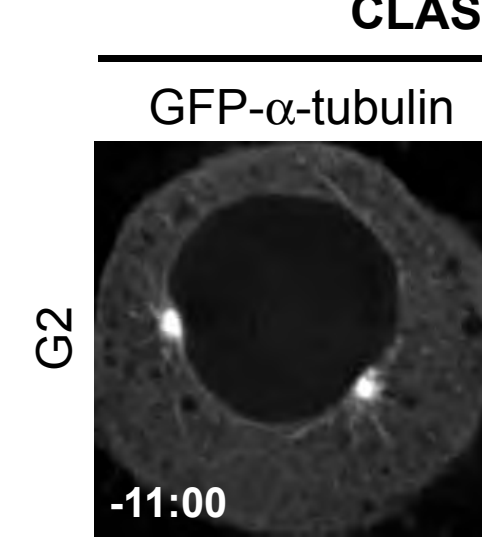

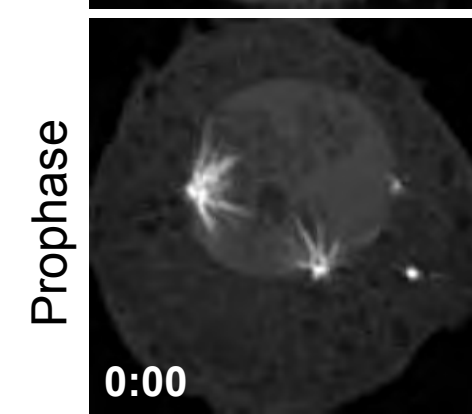

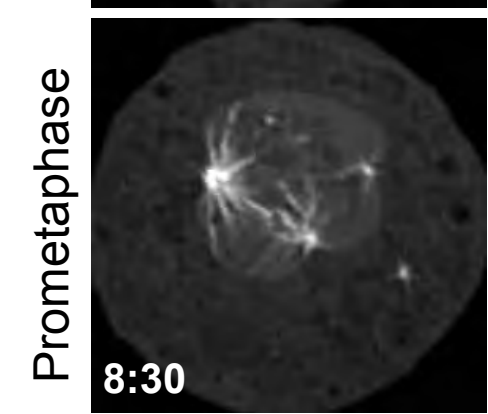

\section{CLASP RNAi}
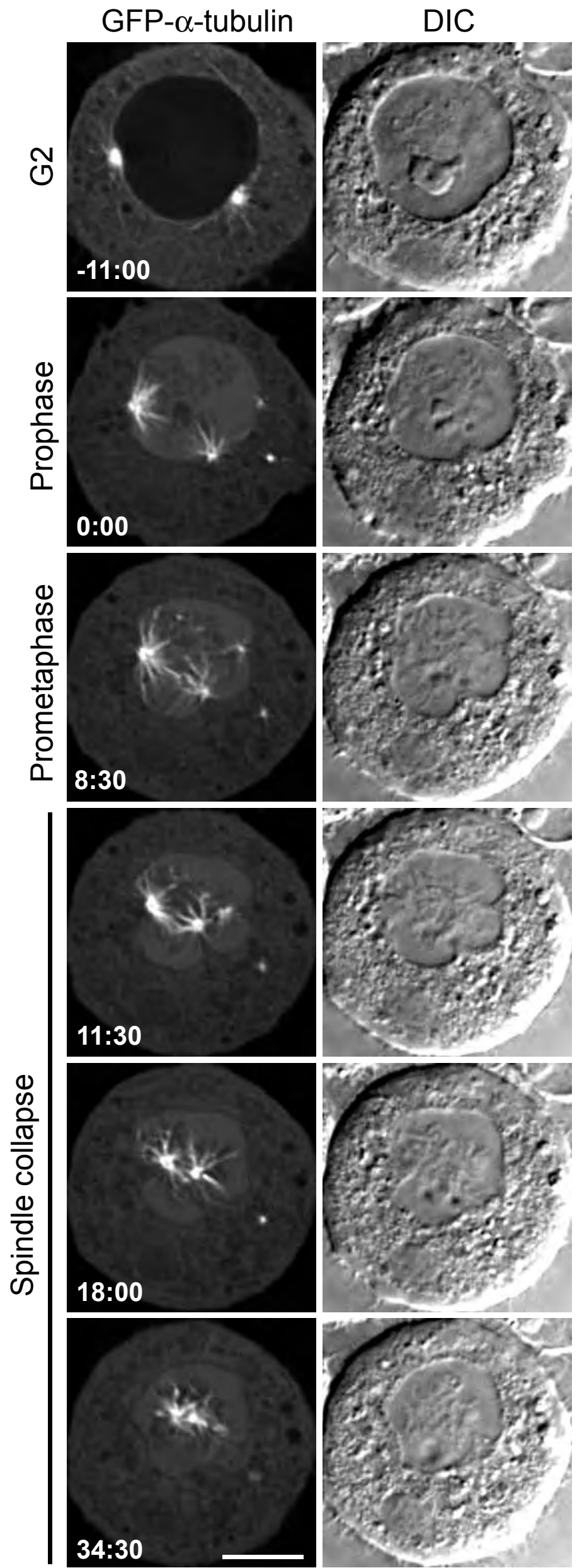

Figure 2 - Pereira et al. 

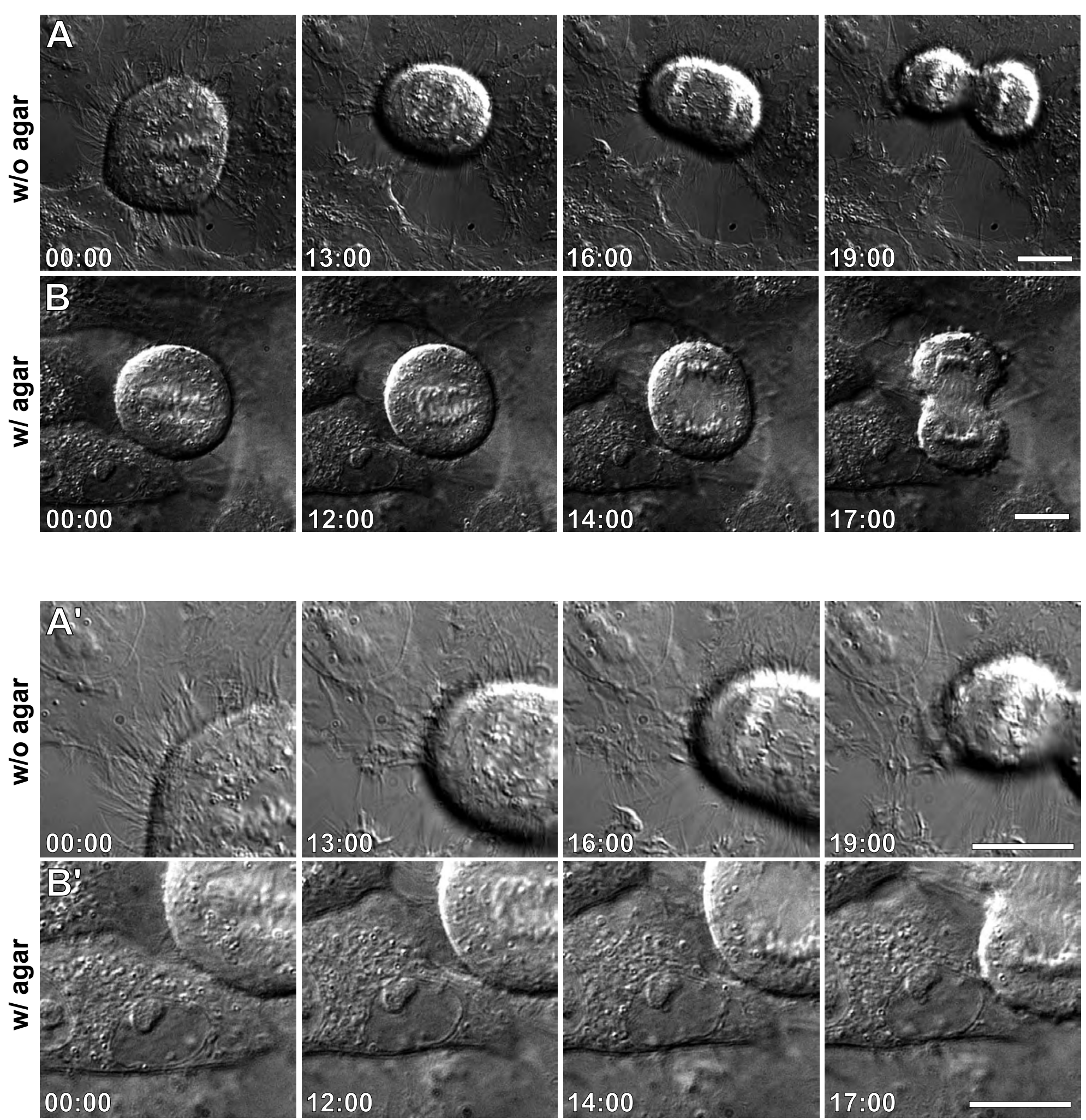

Figure 3 - Pereira et al. 


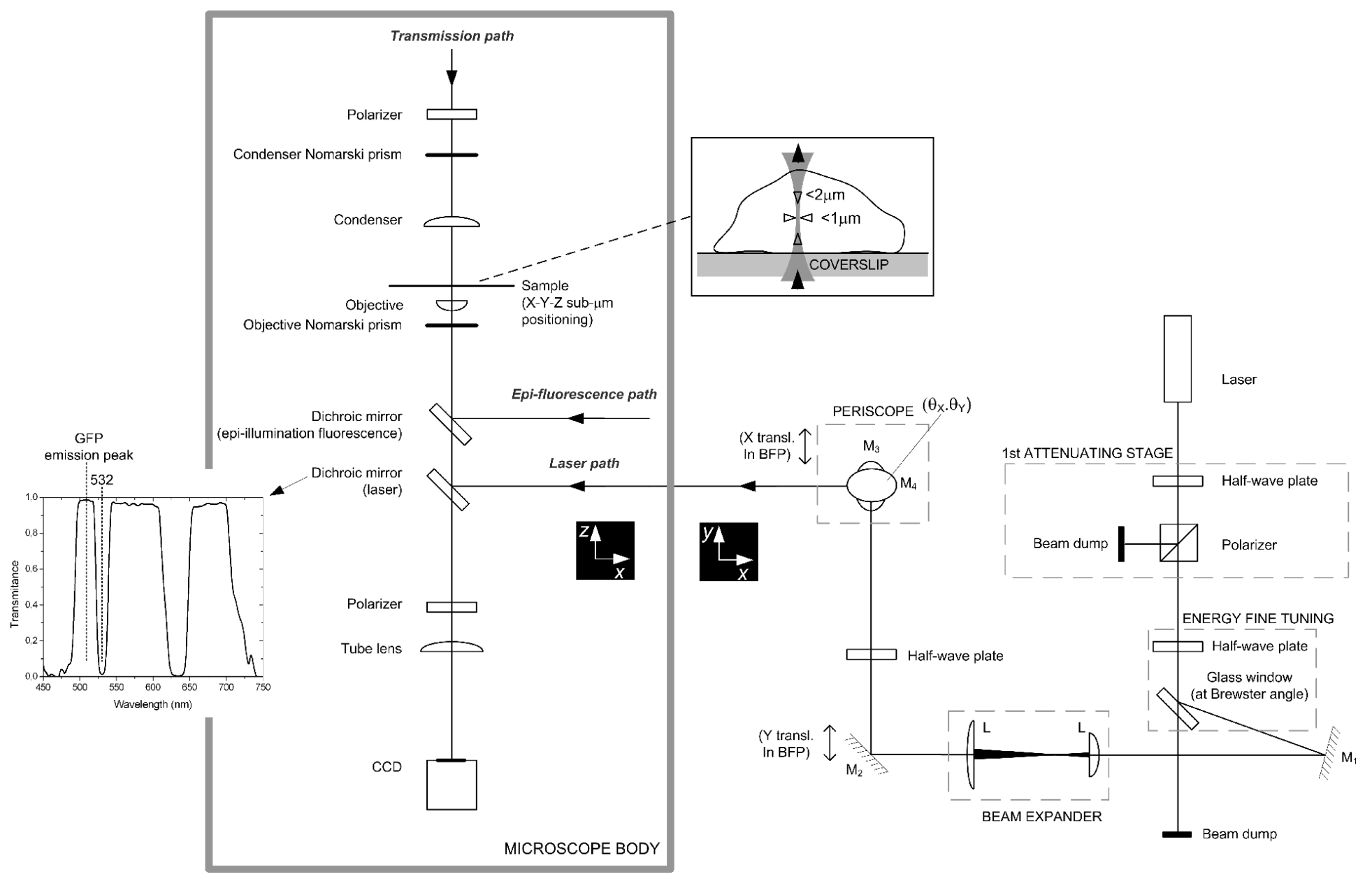

Figure 4 - Pereira et al. 

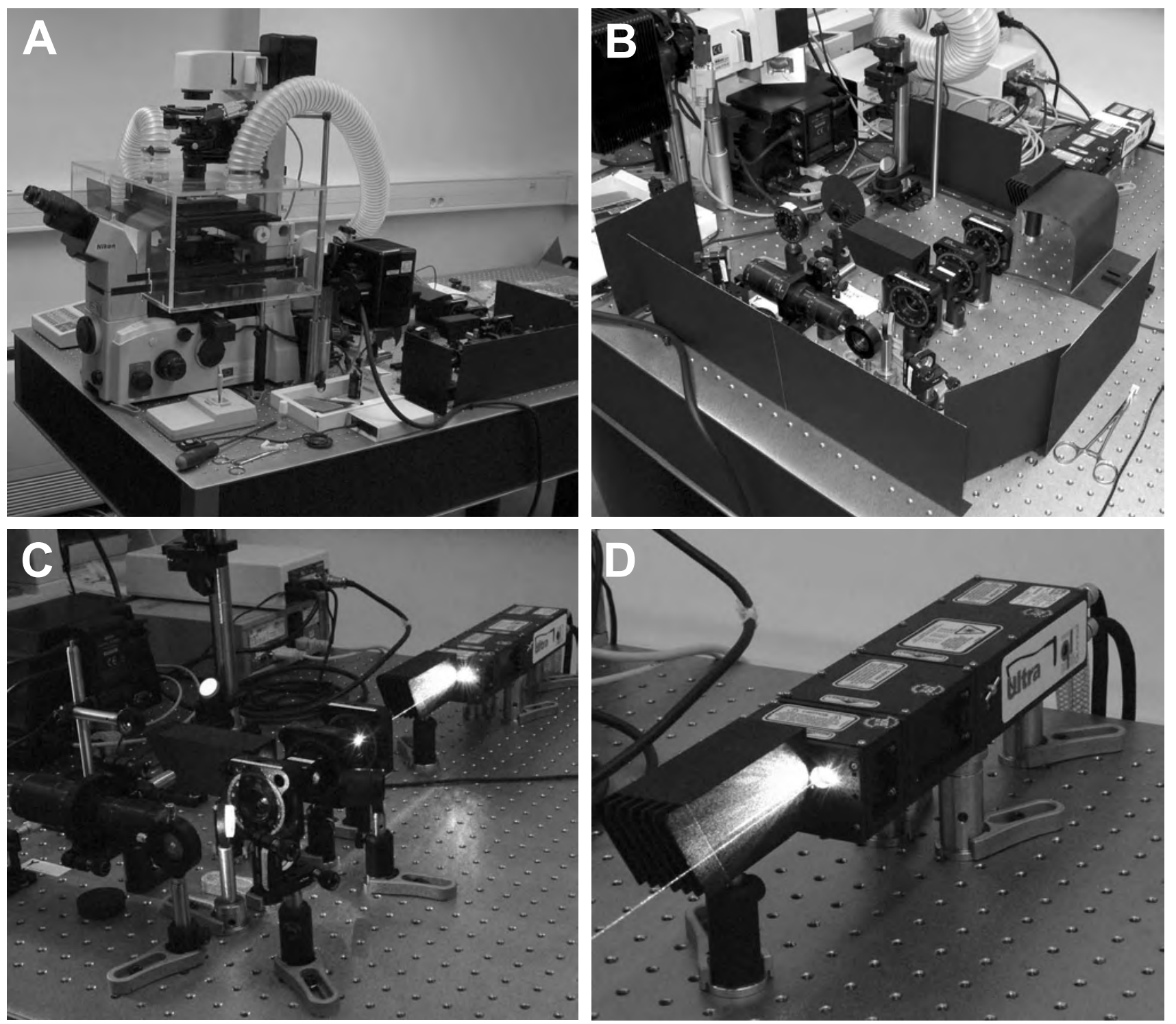

Figure 5 - Pereira et al. 


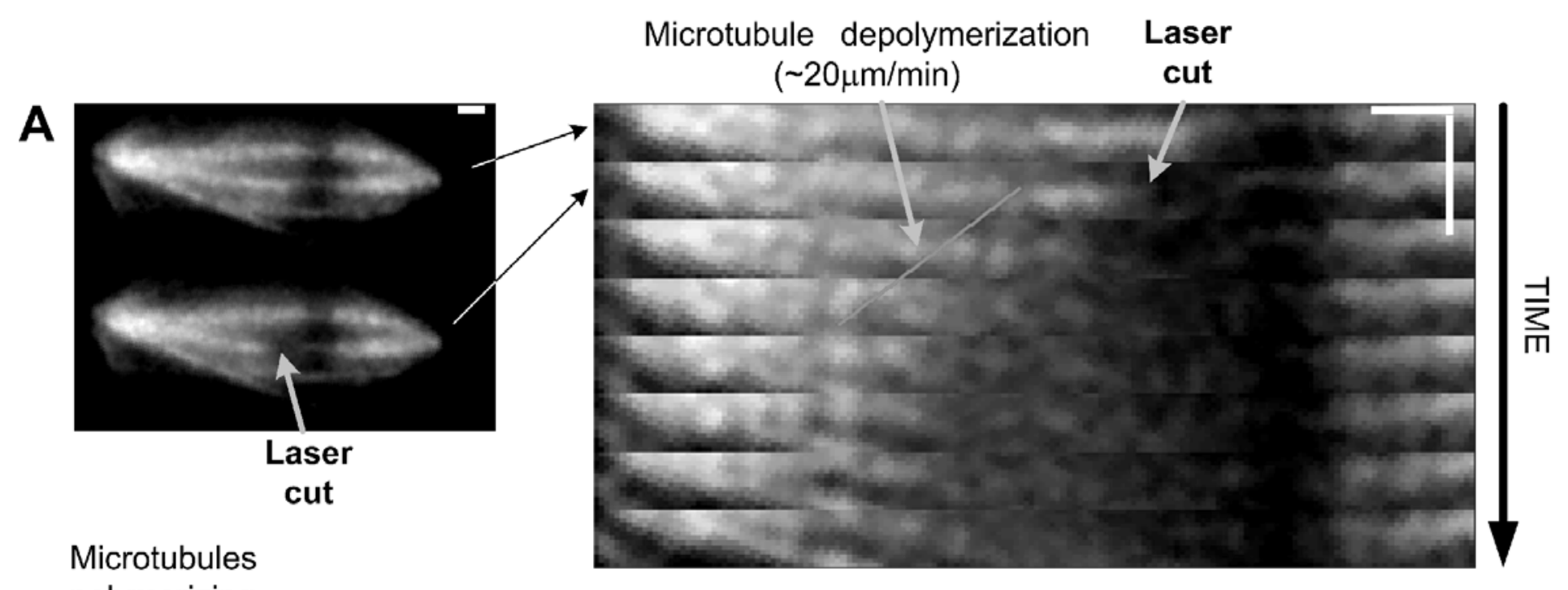

polymerizing from chromosome Laser Depolymerizing

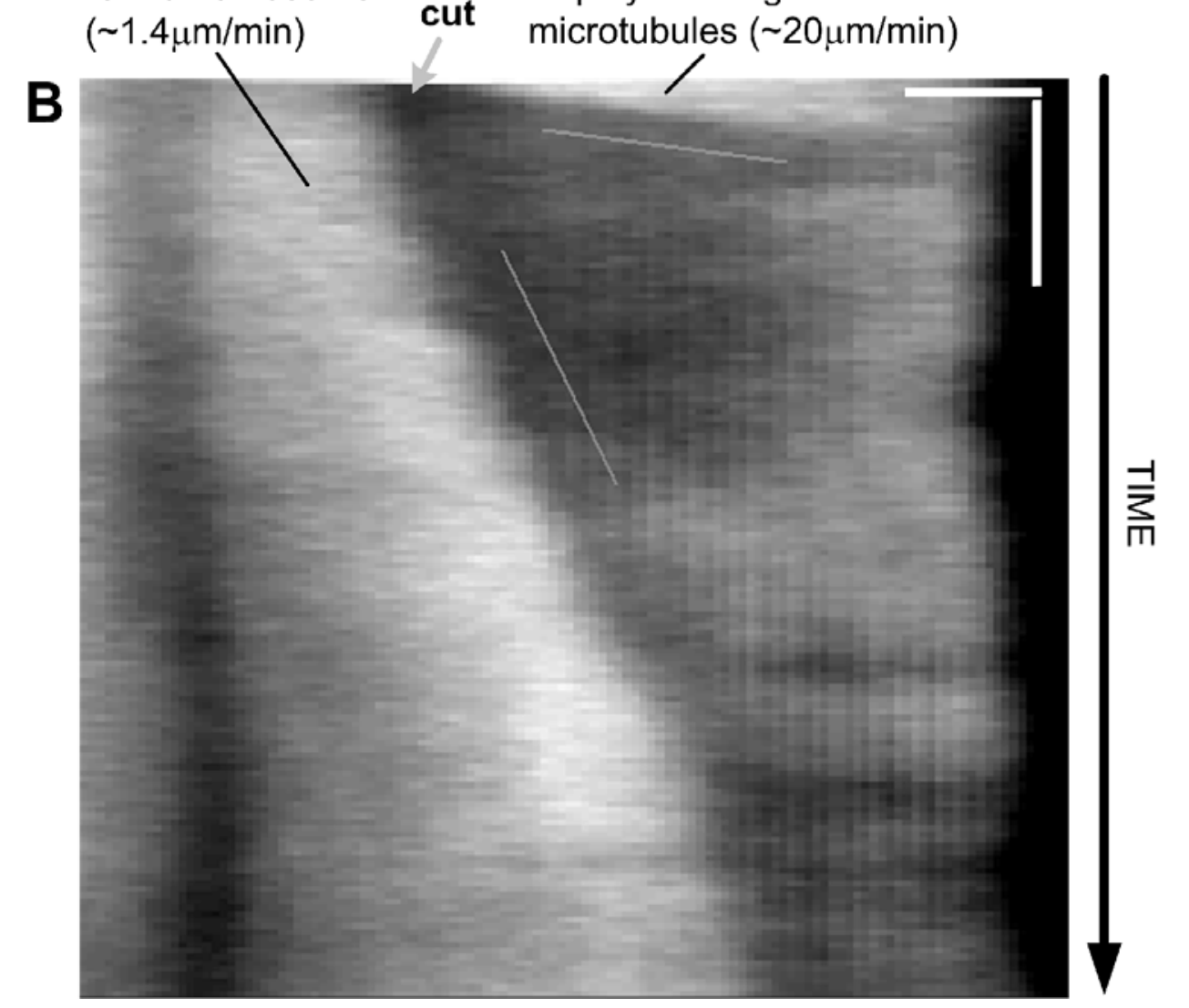

C

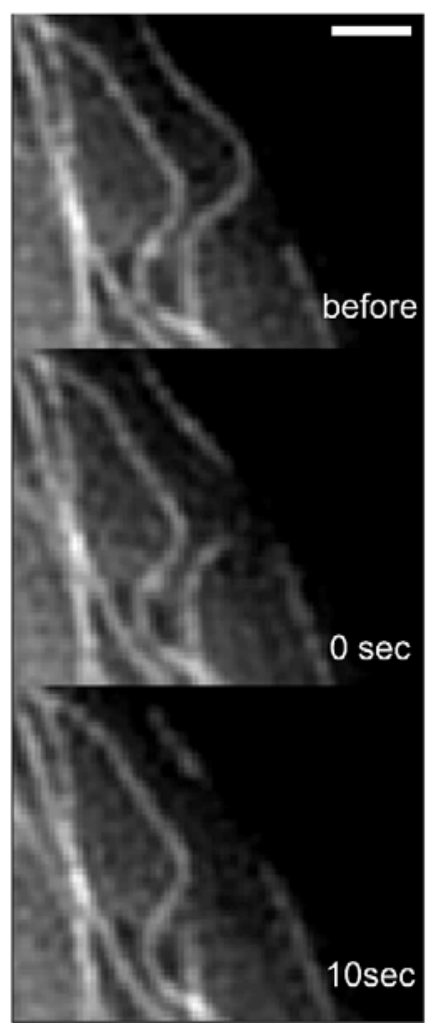

Figure 6 - Pereira et al. 